\title{
Sender Scorecards for the Prevention of Unsolicited Communication
}

\author{
Andreas U. Schmidt and Andreas Leicher \\ Novalyst IT AG \\ Robert-Bosch-Straße 38, 61184 Karben, Germany \\ Email at: http://andreas.schmidt.novalyst.de/
}

\author{
Yogendra Shah, Inhyok Cha and Louis Guccione \\ InterDigital Communications, LLC \\ 781 Third Avenue, King of Prussia, PA 19406 \\ \{yogendra.shah,inhyok.cha,louis.guccione\}@InterDigital.com
}

\begin{abstract}
Sender Scorecards are conceived as a method to enable different IP Multimedia Subsystem (IMS) domains and networks to exchange trustworthy information on sender identities and other relevant information, to allow discrimination between legitimate and unsolicited IMS communication. The approach uses a secure and interoperable scorecard about the sender of IMS calls. Such a scorecard is generated, transported, and evaluated collaboratively between different network domains. Scorecards are used to generate a standardized exchange format for sender and message related information. Depending on the outcome of the scorecard evaluation, the receiving domain can take appropriate actions such as denying or allowing a communication attempt. The present paper proposes a blueprint for an IMS architecture including scorecard elements, based on 3GPP standards. In this context, procedures operating with scorecards for prevention of unsolicited communications are presented.
\end{abstract}

\section{INTRODUCTION}

Unsolicited communication is one of the major problems of digital communication. In the form of email SPAM, it makes up for the bulk of email traffic, but the amount of undesired emails reaching the inbox is, for most users, efficiently curbed by filtering methods. For time-critical comunication over the Internet, in particular Voice-over-IP (VoIP), however, the situation can be worsened significantly, since each incoming communication requires a user action and thus limits the use of automatic filtering. The so-called SPIT (SPAM over Internet Telephony) therefore constitutes a major emerging threat to users and networks. Similar threats are considered to be valid for large-scale networks based on the IP Multimedia Subsystem (IMS), in the context of which it is termed Unsolicited Communication over IMS (UCI), and its prevention is called PUCI. UCI threatens users and networks alike due to the potentially high bandwidth consumption of it. PUCI has been considered by the 3GPP's security working group in a technical report [1] which collects various countermeasures against UCI in the context of IMS system architectures. In [1] 3GPP considers two broad categories of UCI, i.e. the SPAM over IMS (SPIM) and SPIT. This document is the basis on which the present concept paper rests.

The main difference between email spam and UCI is, that an email arrives at the email server before it is accessed by the user. This means, that structure and content of an email can be analyzed at the server before it arrives at the recipient and so email spam can be detected before it disturbs the recipient. As in VoIP and IMS communication scenarios delays of communication establishment are not wished, session establishment messages are forwarded immediately to the recipients. Besides this fact the content of a VoIP call is exchanged immediately after the session is established. In other words if the phone rings it is too late for SPIT prevention and the phone rings immediately after session initiation, while an email can be delayed and even, if it is not delayed, the recipient can decide if he wants to read the email immediately or not. In addition to these aspects another main difference between email spam and UCI is the fact, that the single email itself contains information, that can be used for spam detection. The header fields, for example, may contain information about sender, subject and content of the message. UCI in contradiction is technically indistinguishable from a legitimate communication attempt, as it is initiated and answered with the same set of protocol messages as any other IMS communication.

The following are the most widely used countermeasures against SPIT. Active and passive device fingerprinting, presented in [2], are based on the assumption that knowledge about the type of a User Agent (UA) initiating a call helps identifying a SPIT session initiation attempt. Comparison of header layout and order or the response behavior of a SIP UA with a typical UA are used as criteria. In passive fingerprinting, only the normal protocol behaviour of UA is observed, while active fingerprinting sends probe messages to an UA. Whiteblack- and greylists developed for SPAM mitigation, find their counterparts as SPIT countermeasures [3], [4]. In reputation based mechanisms [5], [6], the callee can set a reputation value for the caller, that marks this caller as spitter or legitimate. The reputation value must be assigned to the identity of the caller and can be used in future session establishment requests. Reputation may feed into greylists. By Turing tests, computational puzzles, and CAPTCHAS [7], [8], a caller is given a challenge that a human can solve easily and that is hard to solve for a machine. Such methods bar legitimate callers from getting an immediate connection, and may be circumvented by using (cheap) human workforce. The idea of payments at risk [9] is to raise costs for SPIT callers while keeping legitimate calls cheap. If user A wants to call user B, he must first send a small amount of money (a micropayment) to user B. When User B accepts the call and confirms that the call is not a SPIT call, the amount 
will be charged back to user A. Given the high-volume of SPIT attempts, micropayments introduce significant cost for large-volume SPIT attacks. Several methods are integrated into comprehensive SPIT mitigation frameworks such as in [10], [11], which can be viewed as a blueprint for the IdentificationMarking-Reaction (IMR) concept of [1].

Practically all methods for SPIT prevention in the literature, as well as the UCI countermeasures in [1], follow the paradigm of perimeter security, i.e., a unilateral defense by the receiving domain. The IMR architecture, which features prominently in [1], integrates multiple of such countermeasures in a 'holistic' approach. As was analysed in [12], [13], perimeter security has principal limitations when applied to SPIT/UCI, due to the lack of trust in any information coming from the sender domain (which can include non-IMS, non-trusted domains), sender UAs, or intermediate nodes. This led us to the idea of a PUCI method relying on inter-domain collaboration and information exchange based on Sender Scorecards (SSC). These PUCI SSC transport trustworthy information that can be used in UCI classification and also supplement other existing countermeasures such as those described earlier in this section. SSC is an orthogonal concept to the traditional mitigation methods, and could be combined with IMR, for instance.

Scoring methods are used extensively in corporate management, as a performance measurement and quality or process improvement instrument, for instance in the form of Balanced Scorecards, introduced in the 1980s [14]. A first approach to use of scoring in SPIT preventions appears in [15]. The present paper further develops this earlier concept and aims to provide a framework to create, manage, and transport UCI score information between domains. Such information can be about sender trustworthiness such as identity and authentication strength, reputation or device security properties, as well as trust properties of intermediate nodes and networks, or message properties, e.g. protocol header information assessment.

In Section II, an architecture for inclusion of scorecard information in an IMS network is sketched. Section III shows basic SSC processing and call flows in a template fashion and proposes an expression format for SSCs. Section IV concludes the paper with an outlook to concrete realisation options.

\section{SENDER SCORECARDS IN IMS ARCHITECTURE}

Introduced as an umbrella scheme for generation, assessment, and operational use of heterogeneous sender information for UCI prevention, PUCI SSCs are handled by an intermediate architectural layer between sender and receiver.

A general scheme to transport sender identity and other information, relevant for UCI detection and prevention across different identifier domains, i.e. IMS and non-IMS networks must fulfill different requirements. It must provide reliable assurance on different properties of the sending user agent device for the receiving domain. However, the establishment, verification and certification of properties can be different between the IMS (and non-IMS) networks. By the use of a standardized scorecard approach, the receiving domain is able to verify received information from the sender domain and
TABLE I

SCORECARD CONTENT EXAMPLE.

\begin{tabular}{|l|l|l|l|}
\hline Category & Claim & Score & Attribute \\
\hline Identification & $\begin{array}{l}\text { Authenticated using } \\
\text { username/password } \\
\text { Can be resolved to a } \\
\text { physical identity }\end{array}$ & 3 & $\begin{array}{l}\text { Verified by } V_{0} \\
\text { at } t_{0} \\
\text { Verified by } V_{1} \\
\text { Is certified by manufac- }\end{array}$ \\
Device & $\begin{array}{l}\text { turer } \\
\text { Verified by } V_{2} \\
\text { Hevice } \\
\text { checked been integrity } \\
\text { Reputation score in } \\
\text { community } X \text { is high }\end{array}$ & 8 & $\begin{array}{l}\text { at } t_{2} \\
\text { Verified by } V_{i} \\
\text { at } t_{i} \\
\text { Verified by } V_{n} \\
\text { at } t_{n}\end{array}$ \\
\hline
\end{tabular}

build its decision on whether to allow or disallow communication to the receiving UA in domain $\mathrm{B}$.

Each SSC is built from multiple entries, with each of them having a single category, a claim which defines the property in this category, a score, which is globally defined and comparable, and an attribute field which keeps track of the state of the claim. Categories and scores must be comparable, e.g. by standardisation and agreements between domain operators. Different claims allow to assert properties on the sender and sending device. Examples are given in Table I. The verification of the claims and the attribute field, reflecting any changes to the state of a claim, play a central role in the concept of the SSCs. The SSC allows the receiving network and UA to assess the level of SPIT/SPAM probability using a standardised format for information exchange. The approach is implementation independent in the sense that the structure is standardised, whereas actual claims and properties will vary depending on the network's and device's capabilities.

\section{A. Scorecard Expression Format}

Technically, several options for the implementation of the SSCs are possible; one example would be a XML scheme which provides the necessary portability across the different domains. The XML scheme could be embedded or complemented by SAML assertions. With the use of SAML assertions it could be possible to implement SSCs in which different claims are verified and signed by different entities. This allows for a heterogeneous approach, in which not one single entity has to verify all claims before issuing the scorecard, but can instead rely on assertions made by other claim verifiers. A further variant would be to define appropriate SAML attributes for the assertions being made in the SSCs which can be grouped into different domains, as shown in Table I.

Each possible expression variant must protect the integrity of the statements being made in the SSC. Such protection is typically achieved by the use of digital signatures. Hence, signatures become an important part of the SSC architecture. The use of asymmetric cryptography introduces the need for a PKI into the overall architecture. However, other means such as keyed HMAC for integrity protection can rely on symmetric shared secrets, which are shared between trusted domains. 


\section{B. Architecture for Scorecards}

The use of SSCs introduces new architecture elements in the network domains which are responsible for the creation and handling of SSCs. Figure 1 gives an overview of the architecture, showing two different operator domains.

SSCs are provided by a scorecard issuer (SC-I) in each domain for the sending devices in this domain. Whenever a device wants to establish an IMS connection, it requests a SSC from SC-I. The device authenticates towards the SC-I, which allows the SC-I to look up if it has previously issued a SSC for this device. If the SC-I stored a SSC for this device it may check if existing data needs to be updated. If no SSC exists, the SC-I can create a new one, by adding claims based on the capabilities of the device and the network domain. The SC-I is responsible for the verification of the claims, before adding them to the SSC. The SC-I could be implemented in different ways, but the simplest form would verify all claims and sign them using an appropriate digital signature method which might need additional infrastructure elements. Depending on the network architecture, even more complex schemes can be employed, e.g. by relying on claim assertions made by other (trusted) third parties, and only verifying a subset of the SSC at the SC-I. The SC-I should then at least provide a timestamp and a signature on the final SSC, allowing for a statement of freshness and authenticity. A further variant would be to include assertions coming from outside the IMS network. If such assertions can be checked by the SC-I, the SC-I can add them as external (non-IMS domain) assertions but provide a signed statement which is verifiable by the receiving domain. The creation and update process for SSCs is bound to the authentication of the sending device for the communication session. The SSC will be uniquely identifiable and the SC-I which issued the card can always be identified. The SC-I then stores the new SSC in the network or, if the device is able to securely store the card, sends the card to the device. In either case, the SSC is sent together with the session initialization message from sender A to a scorecard proxy (SC-P) in the receiving domain.The SC-P then verifies that the receiver is indeed in domain $\mathrm{B}$ and forwards the SSC to a scorecard verifier (SC-V). The SC-V evaluates the SSC, verifies the signature and timestamp applied to it and optionally also verifies the given claims. The result of the SSC validation is then sent to SC-P which then takes a decision on connection establishment based on the outcome of the validation by SC-V and user- or operator-defined policies, such as allowing only communications where SSCs exhibit high scores.

For the generation of SSCs, it must be possible to query a global database of reference scores for the different claims. The score represents the strength of the score in relation to other possible claims in the same category. For example if a user provides his full name and address to the operator and the operator verified this data, this claim will get a higher score than a (disposable) email address, which itself might have a slightly higher score than a mere pseudonymous username. We will refer to this database as SC-DB. The SC-DB is queried

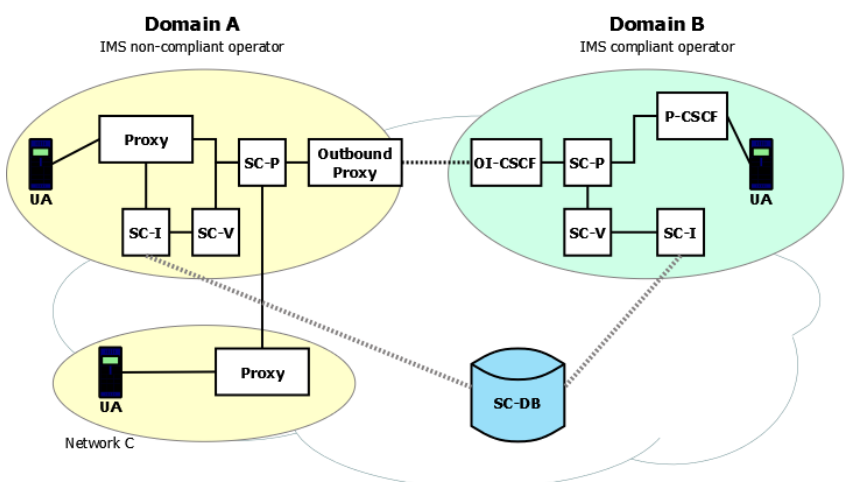

Fig. 1. Basic scorecard architecture.

by the SC-I when new SSCs have to be generated. The SCI looks up the score for every claim and includes the value in the SSC. If possible, the SC-I also verifies the claim, and if SC-I is successful in doing so, SC-I adds a statement on successful verification of the claim including a timestamp of occurred verification in the attribute field for the specific claim in the SSC.

The information in the SSC should be protected for integrity and for those claims which are privacy sensitive also for confidentiality using cryptographic means. The receiving party should not be able to see the privacy sensitive data, but it will be able to see the score, contributing to the overall score of the SSC. Advanced cryptographic methods, such as zeroknowledge proofs could be used to include such information in a privacy protecting manner.

\section{Use of Assertion Providers}

One variant is to use different assertion providers, especially if the SC-I cannot by itself verify all claims. The SC-I tries to verify all yet unverified claims, e.g. by using indicators to responsible verification information providers which are either included in the claims or can be retrieved from the SC-DB. Therefore the SC-I can query different assertion information providers. The referred information providers could for example be an AAA server for additional identity information, device integrity verification servers for device integrity checks, reputation systems, gathering user reputation and behaviour history, etc.. These entities provide assertions on the presented claims and are therefore referred to as Assertion Providers (SC-AP). SC-APs can be situated outside of the domains and can be queried by the SC-Is. A sample of this concept, using distributed databases is shown in figure 2. The SC-I must at least include a notice in the attribute field of the claim, that it retrieved the necessary information from SC-AP, because it cannot be assumed that the receiving domain's $\mathrm{SC}-\mathrm{V}$ is able to verify this assertion from SC-AP. While the general concept uses a central, global database, it could be possible to use different, domain specific SC-DBs for every category as depicted in figure 2. This allows each domain to judge on the score differently and independently.

For example one domain could give a higher score for two- 
factor authenticated identities than another domain. In this case, the scores will have to include an indicator to the SC-DB they have been derived from, allowing the receiving $\mathrm{SC}-\mathrm{V}$ to apply weighting functions to the score.

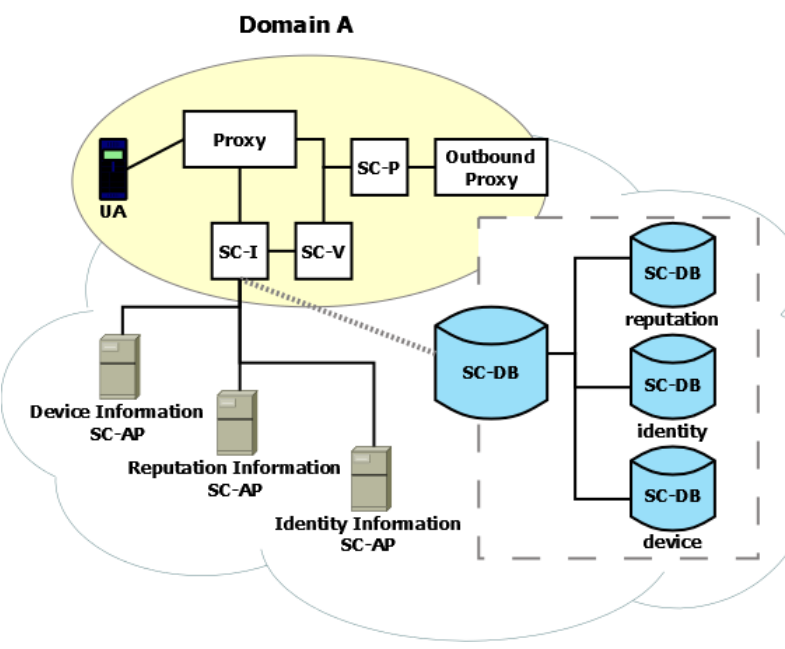

Fig. 2. Scorecard architecture with distributed databases.

\section{SCORECARD PROCESSING}

The verification of the SSC takes place in the SC-V of the receiving network. Since different domains could use different types of claims, the claims are categorized and their score can be looked up by the receiving SC-V using different, independent, global SC-DB, allowing the SC-V to get the scores for individual claims based on the scores stored in the databases. The SSC verifier SC-V is not necessarily involved in the verification of individual claims, such as the evaluation of device integrity measurements or authentication credentials. All claim validation related tasks are performed in the sending network by the respective SC-I upon creation of the SSC. The $\mathrm{SC}-\mathrm{V}$ is intended to check if the SSC is valid, i.e. the SC-V first verifies the freshness using the timestamp and authenticity by verifying the signature. The SC-V further checks if the included claims are compliant to domain/user specific rules, e.g. users might set up rules indicating that they only want to receive messages from devices with an identification score above a treshold value. In addition to that, the SC-V could also be able to verify the trustworthiness of the issuing SC-I in the sender domain, e.g. based on technical means, such as platform integrity verification, or based on other available data such as existing business relationships or the reputation and behaviour of the sender domain.

The calculation of the final score could follow different rules and could even be domain-specific. The calculation method must be standardized and indicated on the SSC. Different options include the use of the lowest score as the total score for a SSC, category-based calculations, where a score is calculated for each category or a weighted calculation using different weights for claim types or categories. The receiving network may also keep network scores for all sending networks, which express the level of trustworthiness the receiver has in the sending domain's SC-I. The individual caller or calling device's SSC from a sending network with an existing network score will be weighted by the network score. Every domain can have individual and non-uniform weighting mechanisms for all other networks. A new sending network, which is not known by the receiver, should start with a network score which is below the lowest network score known to the receiver. Network score can be increased by different mechanisms, such as reputation, agreements between domain operators or certifications of domains.

\section{A. Device Binding of Scorecards}

Instead of issuing a new SSC for every communication attempt being made, the SC-I issues a SSC which is re-usable. Such a SSC requires additional protection in the sense that it cannot be used by another device, such that redistribution to another device is impossible or at least useless when the SSC cannot be used with another device. Furthermore, the SSC should then include at least one timestamp to state freshness of the claims and their verification. One option would be to encrypt the SSC (or parts of it) with a secret which is only known to the sending device.

The device should present reliable evidence of its capabilities that it can store the SSC securely and that the key used for encryption and decryption is protected by the device. The use of existing and standardised security technologies and device profiles enables interoperability of heterogeneous networks, where multiple devices from different vendors with varying hardware (and software) capabilities interact. The device can then decrypt the SSC when initiating a communication session and present the SSC to the receiving domain's SC-P. Such a scheme of re-usable SSCs reduces network traffic for the SC-I but increases the requirements on the device side.

\section{B. Re-use of Scorecards}

If the SSC contains sufficiently strong, verified, identity information, and satisfies the conditions of device binding as detailed above, the SSC provides high assurance about the identity and properties of the party initiating communication. It then has all the information of a credential as is commonly used in Identity Management (IdM) systems. In fact, the SSC is enriched by other information which would be called claims in IdM. It may therefore be natural for the SC-Ps in sending and receiving network to consider and use such Id-enabled SSCs in lieu of access credentials.

A primary use case could be the re-use of SSCs toward various receiving networks. For this, one receiving network's SC-P could be singled out as an identity provider for cardbound identitites, for instance after first receipt and positive assessment of a particular SSC. The SC-P would announce his identity provider (IdP) role for this particular card in a central directory so that other receiving networks called by that user could look up this directory. There, the SC-P of the currently called network could select one card IdP SC-P, for instance the one with freshest assessment information on the 
TABLE II

TYPICAL CALL FLOW.

1) The UA in the sending network A calls the UA in the receiving network. A SSC is sent to SC-P B.

2) SC-P B assesses the SSC and upon positive result...

3) ...forwards the call to the target UA via the Call Session Control Function (CSCF).

4) SC-P B stores the SSC and assessment result in a local SSC Identity Database SC-LIDB. It also publishes its association as IdP to this particular $\mathrm{SC}$ in a public directory SC-IDD. The data sent to the SC-IDD contains (a) the ID of SC-P B, (b) unique ID of the SSC, (c) a lifetime for this IdP association and (d) other data such as integrity/replay protection or metadata.

5) At another time, the same sender UA in network A wants to call a UA in another network C. It sends the same SSC to SC-P C (without knowing the lifetime of that SSC, the sending network may still follow a policy of re-use SSC until failure and only generate a new SSC when the receiving networks rejects the SSC as being too old).

6) SC-P C looks for a SC-IdP association in SC-IDD.

7) SC-P C requests and obtains a SSC identity assertion from SC-P $\mathrm{B}$, for which..

8) ...SC-P B queries his SC-LIDB.

9) The call is forwarded to the target UA.

SSC's claims (meaning that the directory entries bear timestamps) to vouch for the identity and integrity of that caller and obtain an identity assertion. This would avoid locally verifying the claims of that SSC. The resulting mechanism has some similarity to common IdM schemes such as OpenID, on the highest conceptual level. One precondition to that is that a sending network can keep a store of old, but re-usable SSCs. Figure 3 shows a possible call flow, highlighting the general functionality. Security elements may need to be added, in particular replay and integrity protection, where necessary. Table II details the protocol steps, where proxies and gateways are omitted for simplicity.

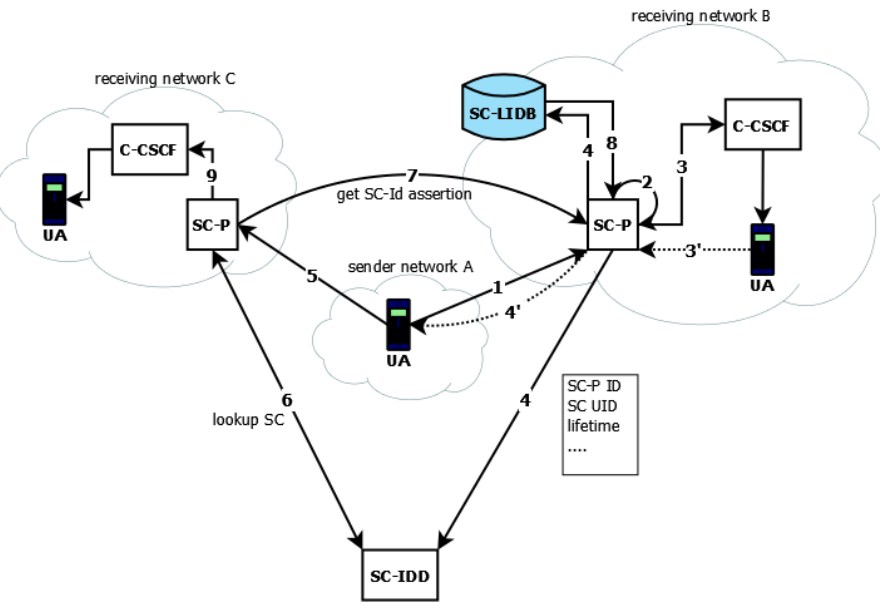

Fig. 3. Protocol flow for scorecard based architecture.

Depending on the availability of network elements, some additional steps may amend the call flow. In a modified step 3', the receiving UA notifies SC-P A that the call was not UC.
SC-P A will then create the SSC IdP binding. In step 4', SC$\mathrm{P}$ B notifies SC-P of sender network A of the lifetime of the IdP commitment made, allowing the sender network to judge when to issue a new SSC or re-use the old one.

\section{Scorecard Categories}

In general it is useful to provide different categories for the claims in the SSCs. As stated in previous sections, there could be independent SC-DBs for every claim category. The contents of the SC-DBs and the categories must be standardized for interoperability between different domains. The SSC concept can easily be extended to include additional categories or subcategories to existing categories.

Identity Scoring includes all information on the sender identity. Several fields can be used in the ID category and as examples would include information on the identity authentication scheme (e.g. username/password, biometrics, twofactor authentication). It can also include information on the assurance level, e.g. that an identity can be resolved to a physical address. Additional inclusion of identity properties, like name, country, company, etc. could be desired for some cases, however, for privacy reasons this information should be protected, using well-known methods, such as zero-knowledge proofs to verify the claims. Some fields might include authentication credentials or references to them. One example could be the inclusion of a signed challenge, if public key crypto authentication is used. Technically, the use of extensible resource identifiers (XRI) allows to create a structured scheme with tags, which are interoperable across domains, and additionally could allow a persistent link to a possibly moving resource. The inclusion of Trusted Sender Identities into the SSC approach is a natural extension to the identity information. 3GPP already identified strong authentication as one key element of PUCI in [1]. Hence, it makes sense to also include the strength of the authentication, as well as additional information on the authentication process in a SSC. Such additional information covers aspects of the device and security features which are used in the authentication procedure. For example an UICC based authentication for mobile devices, performed by the network operator might have an increased level of trust over a SIP digest authentication using a username and password combination which is more prone to phishing and theft. Another authentication mechanism, discussed in 3GPP aims to provide interworking of the GBA protocol and the web-based OpenID protocol [16] to allow for a more secure authentication, which leverages the use of established, MNO based authentication to a general web-based authentication scheme, building on existing security in mobile devices. Another flavour of trusted sender identities can be established when the UA uses HWbased security anchors allowing to assure UA integrity, such that the UA can be expected to behave in the expected manner and is for example not infected with malware, etc. Binding the identification to the assessment of the trustworthiness of the UA is then a key element in establishing trusted identities, relying on the strength of authentication and additional device integrity. By the inclusion of trusted sender identities in a 
SSC, the card will have a higher score and additionally can include references to verifiable trustworthy information. Device Scores are used to characterize devices, by different features such as vendor, device type, such as handset, laptop, software version, device certification, etc. It is further possible to introduce Reputation-Based Scoring into the SSC, which allows establishing a certain score based on a reputation or network of reputations, similar to social-networks or webs of trust. Additionally, the SSC could also include information on the behavior of the caller. Such information cannot be retrieved by the SC-I prior to call initiation and can only be collected by user feedback mechanisms. Such data in the SSC will always be based on historic, long-term data, which must be kept in a database to which the SC-I has access.

\section{CONCLUSION}

In this paper a basic scheme and numerous variants on the use of sender scorecards was presented. Typical SSC content, including critical user and device identification categories were also provided. In presenting the basic architecture for the SSCs, key infrastructure elements were introduced: 1) a SSC issuer (SC-I) which provides a signature, timestamp and queries assertion information providers; 2) a SSC proxy (SC$\mathrm{P})$ which verifies the presence of the receiving device in the receiving domain and makes the final decision on the call; and 3) SSC verifier (SC-V) which verifies the signature and identity claims of the SSC.

The presented concept of SSC can be implemented in addition to the measures for PUCI described in [1]. If a user or domain operator defines target scores for individual claims or claim categories it is possible to implement an automatic score based access control. This could be used to populate blacklists, e.g. by automatically putting all communication attempts with low scores on a blacklist. Another option would be to solve the introduction problem of whitelists by defining minimal scores. If the minimal scores are reached, the communication can be established. In the case of a 'high-score but still UC' the user would then put the caller on the blacklist. If no SSCs are generated in the sending domain (e.g. if no SC-I is present or not implemented), it could be possible to generate SSCs in the receiving domain. The SC-P in the receiving domain would gather different information on the communication (e.g. user feedback, statistical data, etc.) and generate a SSC for this communication. While it is not meant to prevent ongoing SPIT, the SSC could be stored with a device and communication fingerprint in a database. Whenever communication without an associated SSC is received, the SC-P would look up the device/sender fingerprint in the database and check for an existing SSC. If a SSC exists, it will be associated with the communication attempt for the further traffic inside the domain. By integrating SSC functionality in the protocol level, it could be possible to authenticate single transactions, and based on the scores in the SSC, the routers on the way would forward or drop the packets.

Issues attendant relating to the use of SSCs use were also discussed. Of these, SSC re-use is considered to be of particular importance for efficiency. In this context device binding was presented, where sending device secure storage capabilities are úsed to prevent SSC redistribution to other devices. Moreover, a typical protocol call flow for a SSC architecture, which included the described features such as re-use, IdM association, SSC local database storage for future use in possibly other receiving domains, was outlined.

\section{ACKNOWLEDGEMENT}

This work was funded by InterDigital, Inc. Special thanks go to Bob DiFazio for many useful discussions and comments.

\section{REFERENCES}

[1] 3GPP TR 33.937 v9.2.0 "Study of Mechanisms for Protection against Unsolicited Communication for IMS (PUCI)," available at http://3gpp. org/ftp/Specs./html-info/33937.htm. June 2010.

[2] H. Yany, K. Sripanidkulchaiz, H. Zhangy, Z. Shaez and D. Saha, "Incorporating Active Fingerprinting into SPIT Prevention Systems," in Proc. VSW'06. ACM, 2006

[3] M. Hansen, M. Hansen, J. Müller, T. Rohwer, C. Tolkmit and H. Waack, Developing a Legally Compliant Reachability Management System as a Countermeasure against SPIT, in Proc. 3rd Annual VoIP Security Workshop, Berlin, Germany. ACM, 2006.

[4] S. Dritsas, J. Mallios, M. Theoharidou, G. F. Marias, and D. Gritzalis, "Threat analysis of the session initiation protocol regarding spam," in Proc. IEEE Intl. Conf. Performance, Computing, and Communications Conference, 2007. IPCCC 2007., 2007, pp. 426 - 433.

[5] M. Stiemerling S. Niccolini, S. Tartarelli, "Requirements and methods for SPIT identification using feedbacks in SIP," Internet-Draft, 2007. [Online]. Available: http://tools.ietf.org/html/ draft-niccolini-sipping-feedback-spit-03

[6] F. Wang, Y. Mo, B. Huang, "P2P-AVS: P2P Based Cooperative VoIP Spam Filtering," in Proc. IEEE WCNC 2007.

[7] C. Jennings, "Computational Puzzles for SPAM Reduction in SIP," Internet-Draft, 2008. [Online]. Available: http://tools.ietf.org/html/ draft-jennings-sip-hashcash-06

[8] H. Tschofenig, E. Leppanen, S. Niccolini, M. Arumaithurai, "Automated Public Turing Test to Tell Computers and Humans Apart (CAPTCHA) based Robot Challenges for SIP," Internet-draft, 2008. [Online]. Available: http://tools.ietf.org/html/draft-tschofenig-sipping-captcha-01

[9] J. Rosenberg and C. Jennings, "The Session Initiation Protocol (SIP) and Spam," RFC 5039 (Informational), Internet Engineering Task Force, Jan. 2008. [Online]. Available: http://www.ietf.org/rfc/rfc5039.txt

[10] M. Nassar, R. State, O. Festor, Intrusion detection mechanisms for VoIP. In Third annual VoIP security workshop (VSW'06). [Online]. Available: http://arxiv.org/abs/cs.NI/0610109 applications, 2007.

[11] M. Nassar, S. Niccolini, R. State, and T. Ewald, "Holistic voip intrusion detection and prevention system," in IPTComm '07: Proc. 1st Intl. conf. on Principles, systems and applications of IP telecommunications. New York, NY, USA: ACM, 2007, pp. 1-9.

[12] A. U. Schmidt, N. Kuntze, and R. E. Khayari, "Spam over internet telephony and how to deal with it," in Proc. of the 7th annual Conf. Information Security South Africa, H. S. Venter, J. H. P. Eloff, L. Labuschagne, and M. M. Eloff, Eds. Information Security South Africa (ISSA), 2008.

[13] A. U. Schmidt, N. Kuntze, and R. E. Khayari, "Evaluating measures and countermeasures for spam over internet telephony," in ISSE 2008 Securing Electronic Business Processes, N. Pohlmann, H. Reimer, and W. Schneider, Eds. Wiesbaden: Vieweg + Teubner, 2008, pp. 329-340.

[14] Robert S. Kaplan and David P. Norton, "The Balanced Scorecard Measures that Drive Performance." Harvard Business Review, 1992, January-February, pp. 71-79.

[15] D. Wing, S. Niccolini, M. Stiemerling, H. Tschofenig, "Spam Score for SIP," Internet-Draft, 2008. [Online]. Available: http://tools.ietf.org/html/ draft-wing-sipping-spam-score- 02

[16] 3GPP TR 33.924 v9.2.0 "Identity management and 3GPP security interworking; Identity management and Generic Authentication Architecture (GAA) interworking," available at http://ftp.3gpp.org/specs/html-info/ 33924.htm. June 2010. 